EUROPEAN JOURNAL OF PURE AND APPLIED MATHEMATICS

Vol. 14, No. 4, 2021, 1283-1294

ISSN 1307-5543 - ejpam.com

Published by New York Business Global

\title{
A New Spline Method for Solving Linear Two-Point Boundary Value Problems
}

\author{
Ahmed Salem Heilat ${ }^{1, *}$, Hamzeh Zureigat ${ }^{1}$, Ra'ed Hatamleh ${ }^{1}$, Belal Batiha ${ }^{1}$ \\ 1 Department of Mathematics, Jadara University, P.O. Box(733), 21111 Irbid, Jordan
}

\begin{abstract}
In this paper, second order linear two-point boundary value problems are treated using new method based on hybrid cubic B-spline. The values of the free parameter, $\gamma$, are chosen via optimization. The value of the free parameter plays an important role in giving accurate results. Optimization of this parameter is carried out. This method is tested on four examples and a comparison with cubic B-spline, trigonometric cubic B-spline and extended cubic B-spline methods has been carried out. The examples suggest that this method produces more accurate results than the other three methods. The numerical results are presented to illustrate the efficiency of our method.
\end{abstract}

2020 Mathematics Subject Classifications: 34B05, 65L10

Key Words and Phrases: Two-point boundary value problems, Cubic B-spline, Trigonometric cubic B-spline, Hybrid cubic B-spline

\section{Introduction}

Boundary value problems occur in various fields of physics, applied mathematics, chemistry, biology, and engineering. Consider the general form of linear second-order two-point boundary value problem :

$$
\begin{aligned}
u^{\prime \prime}(x)+m(x) u^{\prime}(x)+n(x) u(x) & =r(x), \quad x \in[0,1], \\
u(0)=\beta_{1}, \quad u(1) & =\beta_{2},
\end{aligned}
$$

where $m(x), n(x), r(x) \in C^{0}[0,1]$ and $n(x)<0$ on $[0,1]$. The existence of solution to such problem was studied in $[1,2]$.

Linear two-point boundary value problems have been discussed widely and solved numerically by many authors $[3-9,14,21,24,26]$. Homotopy perturbation method is better than most of the other methods in the literature in giving accurate results with rapid convergence [4]. Variational iteration method is successful in dealing with singular problems.

${ }^{*}$ Corresponding author.

DOI: https://doi.org/10.29020/nybg.ejpam.v14i4.4124

Email addresses: ahmed_heilat@yahoo.com (A. S. Heilat), hamzeh.zu@jadara.edu.jo (H. Zureigat), raedhat@yahoo.com (R. Hatamleh), belalbatiha@gmail.com (B. Batiha) 
Moreover, this method does not need discretization, interpolation and it can derive the exact solution by using one iteration only. This method is also valid for large coefficients [5]. The extended adomian decomposition method is a very effective algorithm which provides promising results with simple calculations [6]. Spectral method gives highly accurate solutions to boundary value problems [7]. Reproducing kernel gives quite accurate and efficient solution for linear fourth-order multi-point boundary value problems [8, 9]. Finite difference method gives highly accurate results only at the chosen knots whereas in some other methods, the results can be obtained at any point in the domain [3, 15].

The aim of this research is to use hybrid cubic B-spline (HCBSM) to solve equation (1). This equation had already been treated using cubic B-spline method (CBSM), trigonometric cubic B-spline method (TCBSM), extended cubic B-spline method (ECBSM), and BS2 methods. ECBSM is by far the efficient spline-based method in producing accurate results [15-18]. Cubic spline is used to obtain the solution at any point in the range [1013]. B-spline interpolation is the efficient to interpolate any smooth functions comparing with finite difference, finite element, finite volume methods [15]. Cubic trigonometric Bspline produces more accurate results compared to cubic B-spline if the problems were trigonometric [16]. The ECBSM have a free parameter $\lambda$, and this parameter is important to give more accurate results. The value of $\lambda$ can be obtained by optimization [17]. BS2 methods give more accurate solution [18]. The generalized nonlinear Klien-Gordon equation and nonlinear two-point BVPs have been treated using HCBSM and the results are promising $[19,25]$. Therefore, HCBSM can be applied to solve equation (1) . The hybrid cubic B-spline also contains a free parameter, $\gamma$.

\section{Hybrid Cubic B-spline}

HCBS is a combination between CBS and TCBS [19]. One free parameter, $\gamma$, is introduced within the basis function. For a finite interval $[0,1]$, suppose that $\left\{x_{i}\right\}_{i=0}^{n}$ is a uniform partition of a finite interval $[0,1]$ with $n \in \mathbb{Z}^{+}$such that $0=x_{0}<x_{1}<\ldots<x_{n}=$ 1. We can extend the partition using

$$
h=\frac{1-0}{n}, \quad x_{0}=0, \quad x_{i}=x_{0}+i h, \quad i \in \mathbb{Z} .
$$

Hybrid cubic B-spline basis function, $H_{i}^{4}(x)$, can be defined as:

$$
H_{i}^{4}(x)=\gamma B_{i}^{4}(x)+(1-\gamma) T_{i}^{4}(x), \quad \gamma \in \mathbb{R} .
$$

where $B_{i}^{4}(x)$ and $T_{i}^{4}(x)$ is a basis functions of cubic B-spline and trigonometric cubic B-spline, respectively $[15,19,20,22]$, as shown in (3)-(4). 


$$
\begin{aligned}
& B_{i}^{4}(x)=\frac{1}{24 h^{4}}\left\{\begin{array}{l}
\left(x-x_{i}\right)^{3}, \quad x \in\left[x_{i}, x_{i+1}\right], \\
h^{3}+3 h^{2}\left(x-x_{i+1}\right)+3 h\left(x-x_{i+1}\right)^{2}-3\left(x-x_{i+1}\right)^{3}, \quad x \in\left[x_{i+1}, x_{i+2}\right], \\
h^{3}+3 h^{2}\left(x_{i+3}-x\right)+3 h\left(x_{i+3}-x\right)^{2}-3\left(x_{i+3}-x\right)^{3}, \quad x \in\left[x_{i+2}, x_{i+3}\right], \\
\left(x_{i+4}-x\right)^{3}, \quad x \in\left[x_{i+3}, x_{i+4}\right] .
\end{array}\right. \\
& T_{i}^{4}(x)=\frac{1}{\kappa} \begin{cases}p^{3}\left(x_{i}\right), \quad x \in\left[x_{i}, x_{i+1}\right], \\
p\left(x_{i}\right)\left[p\left(x_{i}\right) q\left(x_{i+2}\right)+p\left(x_{i+1}\right) q\left(x_{i+3}\right)\right]+p^{2}\left(x_{i+1}\right) q\left(x_{i+4}\right), & x \in\left[x_{i+1}, x_{i+2}\right], \\
q\left(x_{i+4}\right)\left[p\left(x_{i+1}\right) q\left(x_{i+3}\right)+p\left(x_{i+2}\right) q\left(x_{i+4}\right)\right]+p\left(x_{i}\right) q^{2}\left(x_{i+3}\right), & x \in\left[x_{i+2}, x_{i+3}\right], \\
q^{3}\left(x_{i+4}\right), \quad x \in\left[x_{i+3}, x_{i+4}\right],\end{cases} \\
& p\left(x_{i}\right)=\sin \left(\frac{x-x_{i}}{2}\right), \quad q\left(x_{i}\right)=\sin \left(\frac{x_{i}-x}{2}\right), \quad \kappa=\sin \left(\frac{h}{2}\right) \sin (h) \sin \left(\frac{3 h}{2}\right) .
\end{aligned}
$$

If $\gamma=0$, this basis function is equal to cubic trigonometric B-spline basis function and if $\gamma=1$, the basis function becomes B-spline basis function. $H_{i}^{4}(x)$ is a piecewise function of degree 3.

The values of $H_{i}$ and its derivatives $H_{i}^{\prime}, H_{i}^{\prime \prime}$ at the nodal points are tabulated in Table 1 , where

$$
\begin{aligned}
& a_{1}=\frac{\gamma}{6}+\frac{(1-\gamma) \sin ^{2}\left(\frac{h}{2}\right)}{\sin (h) \sin \left(\frac{3 h}{2}\right)}, \quad a_{2}=\frac{4 \gamma}{6}+\frac{2(1-\gamma) \sin \left(\frac{h}{2}\right)}{\sin \left(\frac{3 h}{2}\right)}, \quad a_{3}=\frac{\gamma}{2 h}+\frac{3(1-\gamma)}{4 \sin \left(\frac{3 h}{2}\right)}, \quad a_{4}=\frac{-\gamma}{2 h}-\frac{3(1-\gamma)}{4 \sin \left(\frac{3 h}{2}\right)}, \\
& a_{5}=\frac{\gamma}{h^{2}}+\frac{3(1-\gamma)\left[\sin \left(\frac{h}{2}\right)-2 \sin ^{3}\left(\frac{h}{2}\right)+\sin \left(\frac{3 h}{2}\right)\right]}{8 \sin \left(\frac{h}{2}\right) \sin (h) \sin \left(\frac{3 h}{2}\right)}, \quad a_{6}=\frac{-2 \gamma}{h^{2}}-\frac{3(1-\gamma)\left[\sin (2 h)+2 \sin ^{2}\left(\frac{h}{2}\right) \sin (h)\right]}{4 \sin \left(\frac{h}{2}\right) \sin (h) \sin \left(\frac{3 h}{2}\right)} .
\end{aligned}
$$

Table 1: Coefficient of $H_{i}, H_{i}^{\prime}$ and $H_{i}^{\prime \prime}$

\begin{tabular}{|c|c|c|c|}
\hline$x$ & $x_{i-1}$ & $x_{i}$ & $x_{i+1}$ \\
\hline$H_{i}$ & $a_{1}$ & $a_{2}$ & $a_{1}$ \\
$H_{i}^{\prime}$ & $a_{3}$ & 0 & $a_{4}$ \\
$H_{i}^{\prime \prime}$ & $a_{5}$ & $a_{6}$ & $a_{5}$ \\
\hline
\end{tabular}

From the basis function, an arbitrary spline curve can be generated by the following formula:

$$
S(x)=\sum_{i=-3}^{n-1} C_{i} H_{i}^{4}(x), \quad x \in\left[x_{0}, x_{n}\right], \quad C_{i} \in \mathbb{R} .
$$

There are only four nonzero basis functions, $H_{i-3}^{4}(x), H_{i-2}^{4}(x), H_{i-1}^{4}(x)$, and $H_{i}^{4}(x)$ on the sub-interval $\left[x_{i}, x_{i+1}\right]$. This is due to the local support property of the B-spline basis. At $x_{i}$, there are only three nonzero basis functions; namely, $H_{i-3}^{4}\left(x_{i}\right), H_{i-2}^{4}\left(x_{i}\right)$, and $H_{i-1}^{4}\left(x_{i}\right)$ [19]. So, by finding the first and second derivatives of $S(x)$ and substituting $x_{i}$, we obtain

$$
S\left(x_{i}\right)=A_{1} C_{i-3}+A_{2} C_{i-2}+A_{1} C_{i-1},
$$


A. S. Heilat et al. / Eur. J. Pure Appl. Math, 14 (4) (2021), 1283-1294

$$
\begin{aligned}
S^{\prime}\left(x_{i}\right) & =A_{3} C_{i-3}-A_{3} C_{i-1}, \\
S^{\prime \prime}\left(x_{i}\right) & =A_{4} C_{i-3}+A_{5} C_{i-2}+A_{4} C_{i-1}
\end{aligned}
$$

where

$$
A_{i}=\gamma \sigma_{i}+(1-\gamma) \eta_{i}, \quad \text { for } i=1,2, \ldots, 5
$$

with

$$
\begin{gathered}
\sigma_{1}=\frac{1}{6}, \quad \sigma_{2}=\frac{4}{6}, \quad \sigma_{3}=\frac{-1}{2 h}, \quad \sigma_{4}=\frac{1}{h^{2}}, \quad \sigma_{5}=\frac{-2}{h^{2}}, \\
\eta_{1}=\frac{\kappa_{1}^{2}}{\kappa_{2} \kappa_{3}}, \quad \eta_{2}=\frac{2 \kappa_{1}}{\kappa_{3}}, \quad \eta_{3}=\frac{-3}{4 \kappa_{3}}, \quad \eta_{4}=\frac{3\left(\kappa_{1}-2 \kappa_{1}^{3}+\kappa_{3}\right)}{8 \kappa_{1} \kappa_{2} \kappa_{3}}, \quad \eta_{5}=\frac{-3\left(\kappa_{4}+2 \kappa_{1}^{2} \kappa_{2}\right)}{4 \kappa_{1} \kappa_{2} \kappa_{3}},
\end{gathered}
$$

and

$$
\kappa_{1}=\sin \left(\frac{h}{2}\right), \quad \kappa_{2}=\sin (h), \quad \kappa_{3}=\sin \left(\frac{3 h}{2}\right), \quad \kappa_{4}=\sin (2 h) .
$$

\section{Hybrid Cubic B-spline interpolation method}

In order to solve problem (1), suppose that the solution is $S(x)$, as follows:

$$
\begin{gathered}
S^{\prime \prime}(x)+m(x) S^{\prime}(x)+n(x) S(x)=r(x), \quad x \in[0,1], \\
S(0)=\beta_{1}, \quad S(1)=\beta_{2} .
\end{gathered}
$$

Substituting $x_{i}$ in (12), we have

$$
S^{\prime \prime}\left(x_{i}\right)+m\left(x_{i}\right) S^{\prime}\left(x_{i}\right)+n\left(x_{i}\right) S\left(x_{i}\right)=r\left(x_{i}\right), \quad i=0,1, \ldots, n .
$$

By substituting equations (6) to (8) into (1), we have

$$
\begin{gathered}
\left\{C_{i-3}\left[\gamma \sigma_{4}+(1-\gamma) \eta_{4}\right]+C_{i-2}\left[\gamma \sigma_{5}+(1-\gamma) \eta_{5}\right]+C_{i-1}\left[\gamma \sigma_{4}+(1-\gamma) \eta_{4}\right]\right\} \\
+m\left(x_{i}\right)\left\{C_{i-3}\left[-\gamma \sigma_{3}-(1-\gamma) \eta_{3}\right]+C_{i-1}\left[\gamma \sigma_{3}+(1-\gamma) \eta_{3}\right]\right\} \\
+n\left(x_{i}\right)\left\{C_{i-3}\left[\gamma \sigma_{1}+(1-\gamma) \eta_{1}\right]+C_{i-2}\left[\gamma \sigma_{2}+(1-\gamma) \eta_{2}\right]+C_{i-1}\left[\gamma \sigma_{1}+(1-\gamma) \eta_{1}\right]\right\}=r\left(x_{i}\right) .
\end{gathered}
$$

By collecting the terms that only contain $C_{i-3}, C_{i-2}$, and $C_{i-1}$ from (14), we have

$$
\begin{gathered}
C_{i-3}\left[\gamma \sigma_{4}+(1-\gamma) \eta_{4}-m\left(x_{i}\right)\left(\gamma \sigma_{3}+(1-\gamma) \eta_{3}\right)+n\left(x_{i}\right)\left(\gamma \sigma_{1}+(1-\gamma) \eta_{1}\right)\right] \\
+C_{i-2}\left[\gamma \sigma_{5}+(1-\gamma) \eta_{5}+n\left(x_{i}\right)\left(\gamma \sigma_{2}+(1-\gamma) \eta_{2}\right)\right] \\
+C_{i-1}\left[\gamma \sigma_{4}+(1-\gamma) \eta_{4}+m\left(x_{i}\right)\left(\gamma \sigma_{3}+(1-\gamma) \eta_{3}\right)+n\left(x_{i}\right)\left(\gamma \sigma_{1}+(1-\gamma) \eta_{1}\right)\right]=r\left(x_{i}\right) .
\end{gathered}
$$


Similarly, the boundary conditions can be simplified as the following:

$$
\begin{gathered}
S(0)=S\left(x_{0}\right)=C_{-3}\left[\gamma \sigma_{1}+(1-\gamma) \eta_{1}\right]+C_{-2}\left[\gamma \sigma_{2}+(1-\gamma) \eta_{2}\right]+C_{-1}\left[\gamma \sigma_{1}+(1-\gamma) \eta_{1}\right]=\beta_{1} \\
S(1)=S\left(x_{n}\right)=C_{n-3}\left[\gamma \sigma_{1}+(1-\gamma) \eta_{1}\right]+C_{n-2}\left[\gamma \sigma_{2}+(1-\gamma) \eta_{2}\right]+C_{n-1}\left[\gamma \sigma_{1}+(1-\gamma) \eta_{1}\right]=\beta_{2}
\end{gathered}
$$

We can rearrange (15), (16), and (17) to get a matrix equation in the form of

$$
[A]_{(n+3) \times(n+3)}[C]_{(n+3) \times 1}=[R]_{1 \times(n+3)},
$$

where $C=\left[C_{-3}, C_{-2}, C_{-1}, \ldots, C_{n-1}\right]^{T}$ is the unknown vector, $R=\left[\beta_{1}, r\left(x_{0}\right), r\left(x_{1}\right), \ldots, r\left(x_{n}\right), \beta_{2}\right]^{T}$, and $\mathrm{A}$ is an $(n+3) \times(n+3)$-dimensional matrix given by

$$
A=\left(\begin{array}{ccccccc}
\gamma \sigma_{1}+(1-\gamma) \eta_{1} & \gamma \sigma_{2}+(1-\gamma) \eta_{2} & \gamma \sigma_{1}+(1-\gamma) \eta_{1} & 0 & \ldots & 0 & 0 \\
a_{0}\left(x_{0}\right) & b_{0}\left(x_{0}\right) & c_{0}\left(x_{0}\right) & 0 & \ldots & 0 & 0 \\
0 & a_{1}\left(x_{1}\right) & b_{1}\left(x_{1}\right) & c_{1}\left(x_{1}\right) & 0 & \ldots & 0 \\
\vdots & \vdots & \vdots & \vdots & \vdots & \vdots & \vdots \\
0 & \cdots & 0 & 0 & a_{n}\left(x_{n}\right) & b_{n}\left(x_{n}\right) & c_{n}\left(x_{n}\right) \\
\cdot & \cdot & \cdot & \cdot & \gamma \sigma_{1}+(1-\gamma) \eta_{1} & \gamma \sigma_{2}+(1-\gamma) \eta_{2} & \gamma \sigma_{1}+(1-\gamma) \eta_{1}
\end{array}\right)_{(n+3) \times(n+3)}
$$

The coefficients in the matrix $\mathrm{A}$ are as the following for $i=0,1, \ldots, n$ :

$$
\begin{aligned}
a_{i}\left(x_{i}\right) & =\left[\gamma \sigma_{4}+(1-\gamma) \eta_{4}\right]-m\left(x_{i}\right)\left[\gamma \sigma_{3}+(1-\gamma) \eta_{3}\right]+n\left(x_{i}\right)\left[\gamma \sigma_{1}+(1-\gamma) \eta_{1}\right] \\
b_{i}\left(x_{i}\right) & =\left[\gamma \sigma_{5}+(1-\gamma) \eta_{5}\right]+n\left(x_{i}\right)\left[\gamma \sigma_{2}+(1-\gamma) \eta_{2}\right] \\
c_{i}\left(x_{i}\right) & =\left[\gamma \sigma_{4}+(1-\gamma) \eta_{4}\right]+m\left(x_{i}\right)\left[\gamma \sigma_{3}+(1-\gamma) \eta_{3}\right]+n\left(x_{i}\right)\left[\gamma \sigma_{1}+(1-\gamma) \eta_{1}\right]
\end{aligned}
$$

Therefore, $C$ can be solved by taking

$$
C=A^{-1} R \text {. }
$$

In order to get the approximate analytical solution of equation (1) we can substitute the values of $C_{i}$ in equation (5), for $i=-3,-2, \ldots, n-1$. The numerical solution can be calculated after obtaining the values of $\gamma$ by trial and error [19].

\section{Optimizing the $\gamma$}

The approximate analytical solution is of the form

$$
S(x, \gamma)=\sum_{i=-3}^{n-1} C_{i} H_{i}^{4}(x, \gamma), x \in\left[x_{0}, x_{n}\right], C_{i} \in \mathbb{R} .
$$

where $C_{i}$ 's are obtained by solving a linear system of order $(n+1) \times(n+1) . C_{i}$ 's are functions of $x$ and $\gamma$. The approach used is adopted from [17]. Equation (20) has two free 
parameters, $x$ and $\gamma$. So, $S(x)$ can be written as $S(x, \gamma) . S(x, \gamma)$ is piecewise polynomials with $n$ intervals, as in equation $(21)$. Each $S_{i}(x, \gamma)$, for $i=1,2, \ldots, n-1$ is a polynomials of degree four.

$$
S_{i}(x, \gamma), \quad x \in\left[x_{i}, x_{i+1}\right],
$$

Equation (1) can be written as in Equation (22).

$$
u^{\prime \prime}(x)+m(x) u^{\prime}(x)+n(x) u(x)-r(x) \approx 0 .
$$

Substituting the approximate solutions, $S(x, \gamma)$ and its derivatives into (22), we have

$$
S^{\prime \prime}(x, \gamma)+m(x) S^{\prime}(x, \gamma)+n(x) S(x, \gamma)-r(x) \approx 0
$$

Equation (23) can be written as of error formula. From this equation, we have

$$
D(x, \gamma)=S^{\prime \prime}(x, \gamma)+m(x) S^{\prime}(x, \gamma)+n(x) S(x, \gamma)-r(x), \quad x \in\left[x_{0}, x_{n}\right]
$$

which can be expanded into equation (24).

$$
S_{i}^{\prime \prime}(x, \gamma)+m(x) S_{i}^{\prime}(x, \gamma)+n(x) S_{i}(x, \gamma)-r(x), \quad x \in\left[x_{i}, x_{i+1}\right],
$$

Since equation (24) is piecewise functions with $n$ equations, it is wise to have some representatives from every sub-interval. Suppose we have a sequence, $\left\{x_{i}^{*}\right\}_{i=1}^{n-1}$, where $x_{i}^{*} \in\left[x_{0}, x_{n}\right]$ and $m \in \mathbb{Z}^{+}$such that $x_{i}^{*}=\frac{x_{i}+x_{i+1}}{2}$, for $i=1,2, \ldots, n-1$. Evaluating $D(x, \gamma)$ at $\left\{x_{i}^{*}\right\}_{i=1}^{n-1}$ would produce a sequence of $2 n$ expressions containing $\gamma$,

$$
D\left(x_{i}^{*}, \gamma\right), \quad x \in\left[x_{i}, x_{i+1}\right]
$$

By handling equations (25) as the error at collocation points, the expressions are combined using the two-norm formula resulting equation (26). We hope to minimize $d_{1}(\gamma)$ norm as follows:

$$
d_{1}(\gamma)=\sqrt{\sum_{i=1}^{n-1}\left(D\left(x_{i}^{*}\right), \gamma\right)^{2}}
$$

Also, from equation (26) we can obtain $d_{2}(\gamma)$ which is assumed to be easier to calculate than the former.

$$
d_{2}(\gamma)=\sum_{i=1}^{n-1}\left(D\left(x_{i}^{*}, \gamma\right)\right)^{2}
$$

On the other hand, we can combine the expressions using one-norm formula, as in equation (28).

$$
d_{3}(\gamma)=\sum_{i=1}^{n-1}\left|D\left(x_{i}^{*}, \gamma\right)\right|
$$

This is done to make comparisons between results of $d_{1}(\gamma), d_{2}(\gamma)$, and $d_{3}(\gamma)$ in terms of computational time and accuracy. $d_{3}(\gamma)$ is significantly more simplified that the other 
two. By using Mathematica, we can find the value of $\gamma$ depends on equations (26), (27), and (28) as follows:

$$
\begin{array}{r}
d_{1}(\gamma)=\sqrt{\sum_{i=1}^{n-1}\left(D\left(x_{i}^{*}\right), \gamma\right)^{2}}=0 \\
d_{2}(\gamma)=\sum_{i=1}^{n-1}\left(D\left(x_{i}^{*}, \gamma\right)\right)^{2}=0 \\
d_{3}(\gamma)=\sum_{i=1}^{n-1}\left|D\left(x_{i}^{*}, \gamma\right)\right|=0
\end{array}
$$

Finally, the values of $\gamma$ and $C_{i}$ can be obtained for $i=1,2, \ldots, n-1$. Thus, the solutions for each knot, $x_{i}$, can be obtained from Equation (1).

\section{Numerical Examples and Discussions}

HCBSM was applied on Examples 1-4 with $n=10$ and the results are compared with the analytical solutions. Norms of the results are found numerically by the following formulas:

$$
L_{\infty}=\max _{i=1}^{n-1}\left|S\left(x_{i}\right)-u\left(x_{i}\right)\right|, \quad L_{2}=\sqrt{\sum_{i=1}^{n-1}\left[S\left(x_{i}\right)-u\left(x_{i}\right)\right]^{2}}
$$

Example 1. [15]

$\left\{\begin{array}{l}u^{\prime \prime}(x)-u^{\prime}(x)=-e^{x-1}-1, \quad 0 \leq x \leq 1 \\ u(0)=0, \quad u(1)=0\end{array}\right.$

The analytical solution is $u(x)=x\left(1-e^{x-1}\right)$.

The results for examples 1, 2, 3, and 4 are shown in Tables 2, 3, 4, and 5 and Figures 1, 2, 3 , and 4 , respectively. We found that our method produced much more accurate results than the CBSM [15], TCBSM [16], and ECBSM [23].

Table 2: Absolute errors and norms of CBSM [15], TCBSM [16], ECBSM [23], and HCBSM for Example 1 with $n=10$

\begin{tabular}{|c|c|c|c|c|}
\hline$x$ & CBSM & TCBSM & $\begin{array}{c}\text { ECBSM } \\
(\lambda=2.9375 E-03)\end{array}$ & $\begin{array}{c}\text { HCBSM } \\
(\gamma=1.73125)\end{array}$ \\
\hline 0.1 & $7.531 E-05$ & $1.756 E-04$ & $2.996 E-06$ & $1.568 E-07$ \\
0.2 & $1.439 E-04$ & $3.369 E-04$ & $4.896 E-06$ & $2.264 E-07$ \\
0.3 & $2.031 E-04$ & $4.772 E-04$ & $5.739 E-06$ & $2.062 E-07$ \\
0.4 & $2.499 E-04$ & $5.890 E-04$ & $5.611 E-06$ & $1.022 E-07$ \\
0.5 & $2.803 E-04$ & $6.633 E-04$ & $4.668 E-06$ & $6.743 E-07$ \\
0.6 & $2.900 E-04$ & $6.890 E-04$ & $3.144 E-06$ & $2.696 E-07$ \\
0.7 & $2.736 E-04$ & $6.528 E-04$ & $1.375 E-06$ & $4.527 E-07$ \\
0.8 & $2.249 E-04$ & $5.390 E-04$ & $1.776 E-07$ & $5.425 E-07$ \\
0.9 & $1.366 E-04$ & $3.288 E-04$ & $9.013 E-07$ & $4.369 E-07$ \\
\hline$L_{\infty}$ & $2.900 E-04$ & $6.890 E-04$ & $5.739 E-06$ & $5.425 E-07$ \\
\hline$L_{2}$ & $6.609 E-04$ & $1.568 E-03$ & $1.148 E-05$ & $9.466 E-07$ \\
\hline
\end{tabular}




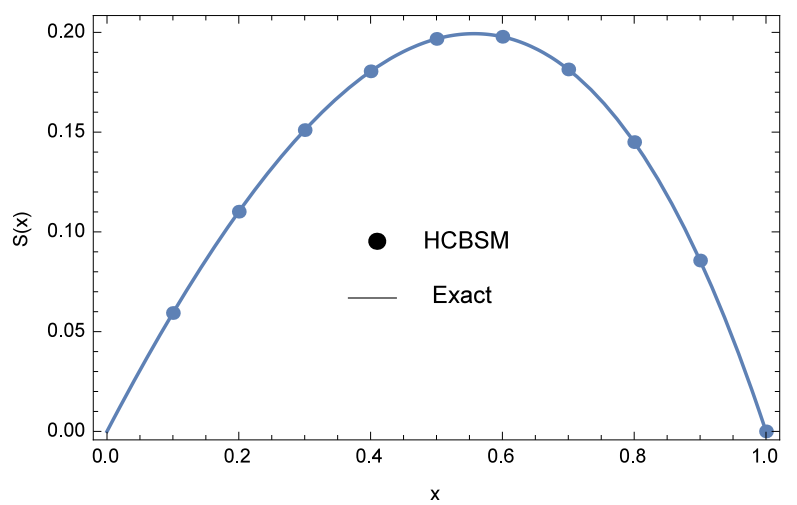

Figure 1: Numerical solution $S(x)$ and exact solution $u(x)$ for Example 1 using HCBSM with $n=10$ and $\gamma=1.73125$

Example 2. [15]

$\left\{\begin{array}{l}u^{\prime \prime}(x)+(x+1) u^{\prime}(x)-2 u(x)=\left(1-x^{2}\right) e^{-x}, \quad 0 \leq x \leq 1 \\ u(0)=-1, \quad u(1)=0\end{array}\right.$

The analytical solution is $u(x)=(x-1) e^{-x}$.

Table 3: Absolute errors and norms of CBSM [15], TCBSM [16], ECBSM [23], and HCBSM for Example 2 with $n=10$

\begin{tabular}{|c|c|c|c|c|}
\hline$x$ & CBSM & TCBSM & $\begin{array}{c}\text { ECBSM } \\
(\lambda=2.9375 E-03)\end{array}$ & $\begin{array}{c}\text { HCBSM } \\
(\gamma=1.6775)\end{array}$ \\
\hline 0.1 & $1.161 E-04$ & $2.922 E-04$ & $1.004 E-06$ & $3.618 E-07$ \\
0.2 & $1.872 E-04$ & $4.686 E-04$ & $3.920 E-07$ & $4.115 E-07$ \\
0.3 & $2.229 E-04$ & $5.547 E-04$ & $1.030 E-06$ & $2.802 E-07$ \\
0.4 & $2.311 E-04$ & $5.719 E-04$ & $2.655 E-06$ & $6.830 E-07$ \\
0.5 & $2.185 E-04$ & $5.376 E-04$ & $4.038 E-06$ & $1.495 E-07$ \\
0.6 & $1.906 E-04$ & $4.661 E-04$ & $4.875 E-06$ & $3.199 E-07$ \\
0.7 & $1.152 E-04$ & $3.687 E-04$ & $4.969 E-06$ & $4.071 E-07$ \\
0.8 & $1.053 E-04$ & $2.543 E-04$ & $4.208 E-06$ & $3.890 E-07$ \\
0.9 & $5.406 E-05$ & $1.297 E-04$ & $2.551 E-06$ & $2.544 E-07$ \\
\hline$L_{\infty}$ & $2.311 E-04$ & $5.719 E-04$ & $4.969 E-06$ & $4.071 E-07$ \\
\hline$L_{2}$ & $5.222 E-04$ & $1.290 E-03$ & $9.912 E-06$ & $9.433 E-07$ \\
\hline
\end{tabular}

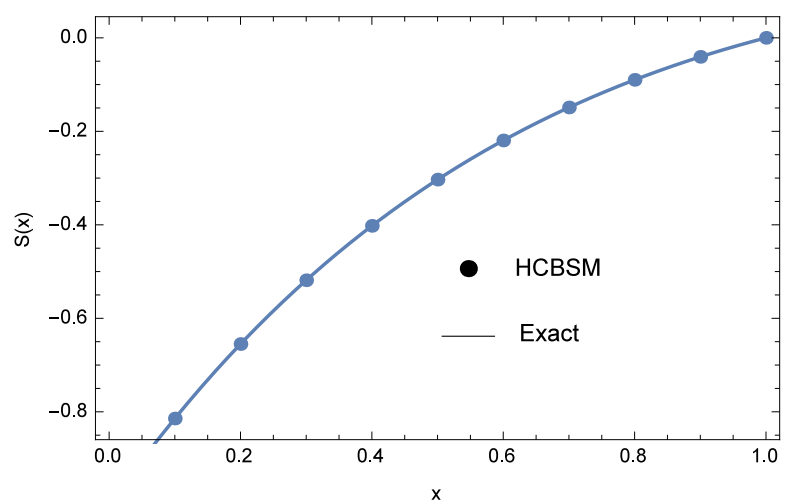

Figure 2: Numerical solution $S(x)$ and exact solution $u(x)$ for Example 2 using HCBSM with $n=10$ and $\gamma=1.6775$ 
Example 3. [2]

$$
\left\{\begin{array}{l}
u^{\prime \prime}(x)-\pi^{2} u(x)=-2 \pi^{2} \sin (\pi x), \quad 0 \leq x \leq 1 \\
u(0)=0, \quad u(1)=0
\end{array}\right.
$$

The analytical solution is $u(x)=\sin (\pi x)$.

Table 4: Absolute errors and norms of CBSM [15], TCBSM [16], ECBSM [23], and HCBSM for Example 3 with $n=10$

\begin{tabular}{|c|c|c|c|c|}
\hline$x$ & CBSM & TCBSM & $\begin{array}{c}\text { ECBSM } \\
(\lambda=-1.6500 E-02)\end{array}$ & $\begin{array}{c}\text { HCBSM } \\
(\gamma=-3.0919)\end{array}$ \\
\hline 0.1 & $1.270 E-03$ & $9.568 E-04$ & $2.769 E-07$ & $5.184 E-09$ \\
0.2 & $2.415 E-03$ & $1.820 E-03$ & $5.267 E-07$ & $9.861 E-09$ \\
0.3 & $3.324 E-03$ & $2.505 E-03$ & $7.249 E-07$ & $1.357 E-08$ \\
0.4 & $3.908 E-03$ & $2.945 E-03$ & $8.522 E-07$ & $1.596 E-08$ \\
0.5 & $4.109 E-03$ & $3.096 E-03$ & $8.960 E-07$ & $1.678 E-08$ \\
0.6 & $3.908 E-03$ & $2.945 E-03$ & $8.522 E-07$ & $1.596 E-08$ \\
0.7 & $3.324 E-03$ & $2.505 E-03$ & $7.249 E-07$ & $1.357 E-08$ \\
0.8 & $2.415 E-03$ & $1.820 E-03$ & $5.267 E-07$ & $9.862 E-09$ \\
0.9 & $1.270 E-03$ & $9.568 E-04$ & $2.769 E-07$ & $5.182 E-09$ \\
\hline$L_{\infty}$ & $4.109 E-03$ & $3.096 E-03$ & $8.960 E-07$ & $1.678 E-08$ \\
\hline$L_{2}$ & $9.188 E-03$ & $6.923 E-03$ & $2.004 E-06$ & $3.751 E-08$ \\
\hline
\end{tabular}

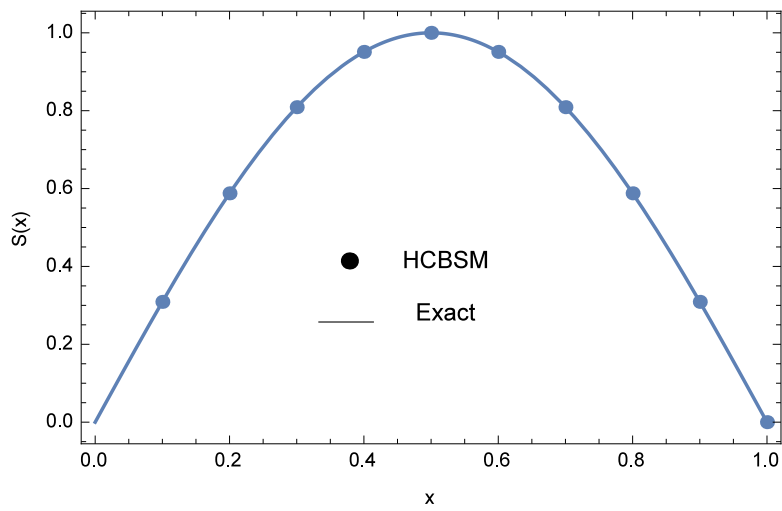

Figure 3: Numerical solution $S(x)$ and exact solution $u(x)$ for Example 3 using HCBSM with $n=10$ and $\gamma=-3.0919$

Example 4. [16]

$$
\left\{\begin{array}{l}
u^{\prime \prime}(x)-u(x)=0, \quad 0 \leq x \leq 1 \\
u(0)=0, \quad u(1)=\sinh (1)
\end{array}\right.
$$

The analytical solution is $u(x)=\sinh (x)$. 
Table 5: Absolute errors and norms of CBSM [15], TCBSM [16], ECBSM [23], and HCBSM for Example 4 with $n=10$

\begin{tabular}{|c|c|c|c|c|}
\hline$x$ & CBSM & TCBSM & $\begin{array}{c}\text { ECBSM } \\
(\lambda=1.6875 E-03)\end{array}$ & $\begin{array}{c}\text { HCBSM } \\
(\gamma=1.32475)\end{array}$ \\
\hline 0.1 & $1.294 E-05$ & $5.269 E-05$ & $1.660 E-07$ & $7.773 E-10$ \\
0.2 & $2.518 E-05$ & $1.025 E-04$ & $3.230 E-07$ & $1.512 E-09$ \\
0.3 & $3.598 E-05$ & $1.465 E-04$ & $4.615 E-07$ & $2.161 E-09$ \\
0.4 & $4.460 E-05$ & $1.816 E-04$ & $5.721 E-07$ & $2.679 E-09$ \\
0.5 & $5.023 E-05$ & $2.045 E-04$ & $6.444 E-07$ & $3.017 E-09$ \\
0.6 & $5.201 E-05$ & $2.118 E-04$ & $6.672 E-07$ & $3.124 E-09$ \\
0.7 & $4.899 E-05$ & $1.995 E-04$ & $6.284 E-07$ & $2.942 E-09$ \\
0.8 & $4.012 E-05$ & $1.634 E-04$ & $5.146 E-07$ & $2.409 E-09$ \\
0.9 & $2.423 E-05$ & $9.866 E-05$ & $3.107 E-07$ & $1.455 E-09$ \\
\hline$L_{\infty}$ & $5.201 E-05$ & $2.118 E-04$ & $6.672 E-07$ & $3.124 E-09$ \\
\hline$L_{2}$ & $1.179 E-04$ & $4.802 E-04$ & $1.513 E-06$ & $7.083 E-09$ \\
\hline
\end{tabular}

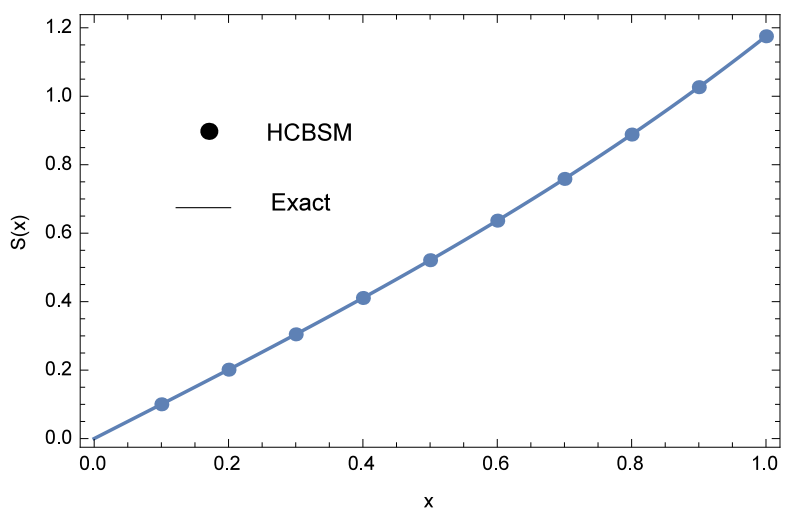

Figure 4: Numerical solution $S(x)$ and exact solution $u(x)$ for Example 4 using HCBSM with $n=10$ and $\gamma=1.32475$

\section{Conclusions}

In this research, a new method for finding approximate solutions for a second-order linear two-point boundary value problems based on hybrid cubic B-spline was proposed. This method is called HCBSM. The values of the free parameter $\gamma$ have influence on the accuracy of our method. This method improved the accuracy of its predecessors; CBM, TCBSM, and ECBSM and produced more accurate results than other spline methods.

\section{References}

[1] Blum, E. K. (1972). Numerical analysis and computation theory and practice.

[2] Burden, R. L. (1985). JD Faires Numerical Analysis. Boston: Brooks-Cole. Pub., pp. 672-674.

[3] Fang, Q., Tsuchiya, T., and Yamamoto, T. (2002). Finite difference, finite element and finite volume methods applied to two-point boundary value problems. Journal of Computational and Applied Mathematics, 139(1), 9-19. 
[4] Chun, C. and Sakthivel, R. (2010). Homotopy perturbation technique for solving twopoint boundary value problems-comparison with other methods. Computer physics Communications, 181(6), 1021-1024.

[5] Lu, J. (2007). Variational iteration method for solving two-point boundary value problems. Journal of Computational and Applied Mathematics, 207(1), 92-95.

[6] Jang, B. (2008). Two-point boundary value problems by the extended Adomian decomposition method. Journal of Computational and Applied Mathematics, 219(1), 253-262.

[7] Costabile, F. and Napoli, A. (2016). A new spectral method for a class of linear boundary value problems. Journal of Computational and Applied Mathematics, 292, 392-341.

[8] Li, Z. Y., Wang, Y. L., Tan, F. G., Wan, X. H., Yu, H., and Duan, J. S. (2015). Solving a class of linear nonlocal boundary value problems using the reproducing kernel. Applied Mathematics and Computation, 265, 1098-1105.

[9] Geng, F. Z., and Qian, S. P. (2014). A new reproducing kernel method for linear nonlocal boundary value problems. Applied Mathematics and Computation, 248, 421425 .

[10] Albasiny, E. L., and Hoskins, W. D. (1969). Cubic spline solutions to two-point boundary value problems. The Computer Journal, 12(2), 151-153.

[11] Fyfe, D. J. (1969). The use of cubic splines in the solution of two-point boundary value problems. The Computer Journal, 12(2), 188-192.

[12] Al-Said, E. A. (1998). Cubic spline method for solving two-point boundary-value problems. Korean Journal of Computitional and Applied Mathematics, 5(3), 669-680.

[13] Khan, A. (2004). Parametric cubic spline solution of two point boundary value problems. Applied Mathematics and Computation, 154(1), 175-182.

[14] Abukhaled, M. (2017). Green's function iterative method for solving a class of boundary value problems arising in heat transfer. Appl. Math. Inf. Sci, 11(1), 229-234.

[15] Caglar, H., Caglar, N., and Elfaituri, K. (2006). B-spline interpolation compared with finite difference, finite element and finite volume methods which applied to two-point boundary value problems. Applied Mathematics and Computation, 175(1), 72-79.

[16] Hamid, N. N., Majid, A. A., and Ismail, A. I. M. (2010). Cubic trigonometric B-spline applied to linear two-point boundary value problems of order two. World Academic of Science, Engineering and Technology, 47, 478-803. 
[17] Heilat, A. S., Hamid, N. N. A., and Ismail, A. I. M.(2016). Extended cubic B-spline method for solving a linear system of second-order boundary value problems. SpringerPlus, 5(1), 1314.

[18] Manni, C., Mazzia, F., Sestini, A., and Speleers, H. (2015). BS2 methods for semilinear second order boundary value problems. Applied Mathematics and Computation, 255, 147-156.

[19] Mat Zin, S., Abd Majid, A., Ismail, A. I. M., and Abbas, M. (2014). Application of Hybrid Cubic B-Spline Collocation Approach for Solving a Generalized Nonlinear Klien-Gordon Equation. Mathematical Problems in Engineering, 2014.

[20] Abbas, M., Majid, A. A., Ismail, A. I. M., and Rashid, A. (2014). The application of cubic trigonometric B-spline to the numerical solution of the hyperbolic problems. Applied Mathematics and Computation, 239, 74-88.

[21] He, J. H., and Ji, F. Y. (2019). Taylor series solution for Lane-Emden equation. Journal of Mathematical Chemistry, 57(8), 1932-1934.

[22] Abd Hamid NN (2010) Splines for linear two-point boundary value problems. MSc thesis, Universiti Sains Malaysia.

[23] Hamid, N. N. A., Majid, A. A., and Ismail, A. I. M. (2011). Extended cubic B-spline method for linear two-point boundary value problems. Sains Malaysiana, 40(11), 12851290 .

[24] UshaRani, R., Rajendran, L., Abukhaled, M. (2021). Approximations for the concentration and effectiveness factor in porous catalysts of arbitrary shape: Taylor Series and Akbari-Ganji's methods. Mathematical Modelling of Engineering Problems, Vol. 8, No. 4, pp. 527-537.

[25] Heilat, A. S., and Ismail, A. M. (2016). Hybrid cubic b-spline for solving Non-linear two-point boundary value problems. International Journal of Pure and Applied Mathematics, 110(2), 369-381.

[26] Hatamleh, R., and Zolotarev, V. A. (2015). On Model Representations of NonSelfadjoint Operators with Infinitely Dimensional Imaginary Component. Journal of Mathematical Physics, Analysis, Geometry 11(2) ,174-186. 